\title{
Burden of Psychological Issues in Young, Adults and Parents Globally and in India due to COVID
}

\author{
Review Article
}

Volume 2 Issue 2- 2021

\author{
Author Details \\ Nirmal Surya ${ }^{1 *}$, Balaji Patil ${ }^{2}$ \\ ${ }^{1}$ Neurologist, India \\ ${ }^{2}$ Head Medical Affairs, Eisai Pharmaceuticals India Pvt Ltd, India \\ *Corresponding author
}

Nirmal Surya Neurologist, Founder Trustee \& Chairman, Epilepsy Foundation, Mumbai, Maharashtra, India

Article History

Received: April 21, 2021 Accepted: June 19, 2021 Published: June 25, 2021

\begin{abstract}
The coronavirus disease (COVID-19) caused by the severe acute respiratory syndrome coronavirus (SARS-CoV-2) was first detected in December 2019 in Wuhan, China, and has since spread to several provinces in China. India had 15.3 million cases as of April 21, 2021, with 13.1 million cases recovered; however, 181 thousand patients died as a result of the covid. The government enforced a full lockdown across the country to break the chain of Covid infections. People became isolated from society as a result of the lockdown. They limited access to the healthcare system, as well as schools, universities, and other social institutions. The new normal was difficult for people to adjust to, the health-care system was on the verge of collapsing, and the fear of COVID caused widespread anxiety and mental health issues.

According to the recent study published in Lancet on November 9, 18 percent of COVID-19 patients developed a mental health issue - like depression, anxiety, or dementia - within 3 months of diagnosis. Their risk was doubled compared to people who didn't have COVID-19. In the study, authors examined whether a diagnosis of COVID-19 was associated with increased rates of subsequent psychiatric diagnoses, and whether patients with a history of psychiatric illness are at a higher risk of being diagnosed with COVID-19, using data from 69 million people, 62354 of whom have been diagnosed with COVID-19.
\end{abstract}

\section{Introduction}

According to the findings, as compared to six other health incidents, such as influenza, respiratory tract infections, skin infection, cholelithiasis, urolithiasis, and fracture of a large bone, diagnosis of COVID-19 was associated with an increased occurrence of a first psychiatric diagnosis in the intervening 14 to 90 days. Anxiety disorders, insomnia, and dementia were the most dangerous. In the 14 to 90 days following COVID-19 diagnosis, 181 percent (95 percent CI 176-186) of people received a psychiatric diagnosis, with $58 \%$ (5264) of those receiving a first diagnosis. In people older than 65 , the occurrence of a first diagnosis of dementia in the 14 to 90 days after COVID-19 diagnosis was $16 \%$ (95 percent CI 12-21) [1].

\section{COVID and Young People}

Young adults have faced a variety of pandemic-related effects, such as university closures and lost jobs, which may have contributed to their poor mental health. During the pandemic, a higher-than-average proportion of young adults (ages 18-24) experience anxiety and/or depressive symptoms (56 percent). Young adults are more likely than other adults to disclose drug use (25\%vs. $13 \%)$ and suicidal thoughts ( $26 \%$ vs. $11 \%)$ [2]. Young adults were also at high risk of poor mental health and drug use disorder prior to the pandemic, but many did not receive care.

Many young adults have experienced heightened anxiety, depression, sleep disturbances, and suicidal thoughts as a result of the pandemic. They've also dealt with a slew of pandemic-related effects, including university closures, shifts to remote jobs, and loss of income or employment, all of which could wreak havoc on their mental health. In comparison to older adults, a substantial share of young adults (ages 18-24) have recorded signs of anxiety and/or depressive disorder since the pandemic-56 percent as of December 2020 - according to Kaiser Family Foundation study [2].

\section{COVID and Adults}

Many people around the world have lost their jobs or income as a result of the pandemic, which has had a negative impact on their 
mental health. Adults who lost a household job during the pandemic had consistently higher rates of anxiety and/or depressive illness symptoms than adults who did not lose a household job $(53 \%$ vs. $32 \%$, respectively; Figure 1). Similarly, the December KFF Health
Tracking Poll found that households suffering income or work loss are substantially more likely to say the coronavirus epidemic has negatively affected their mental health.

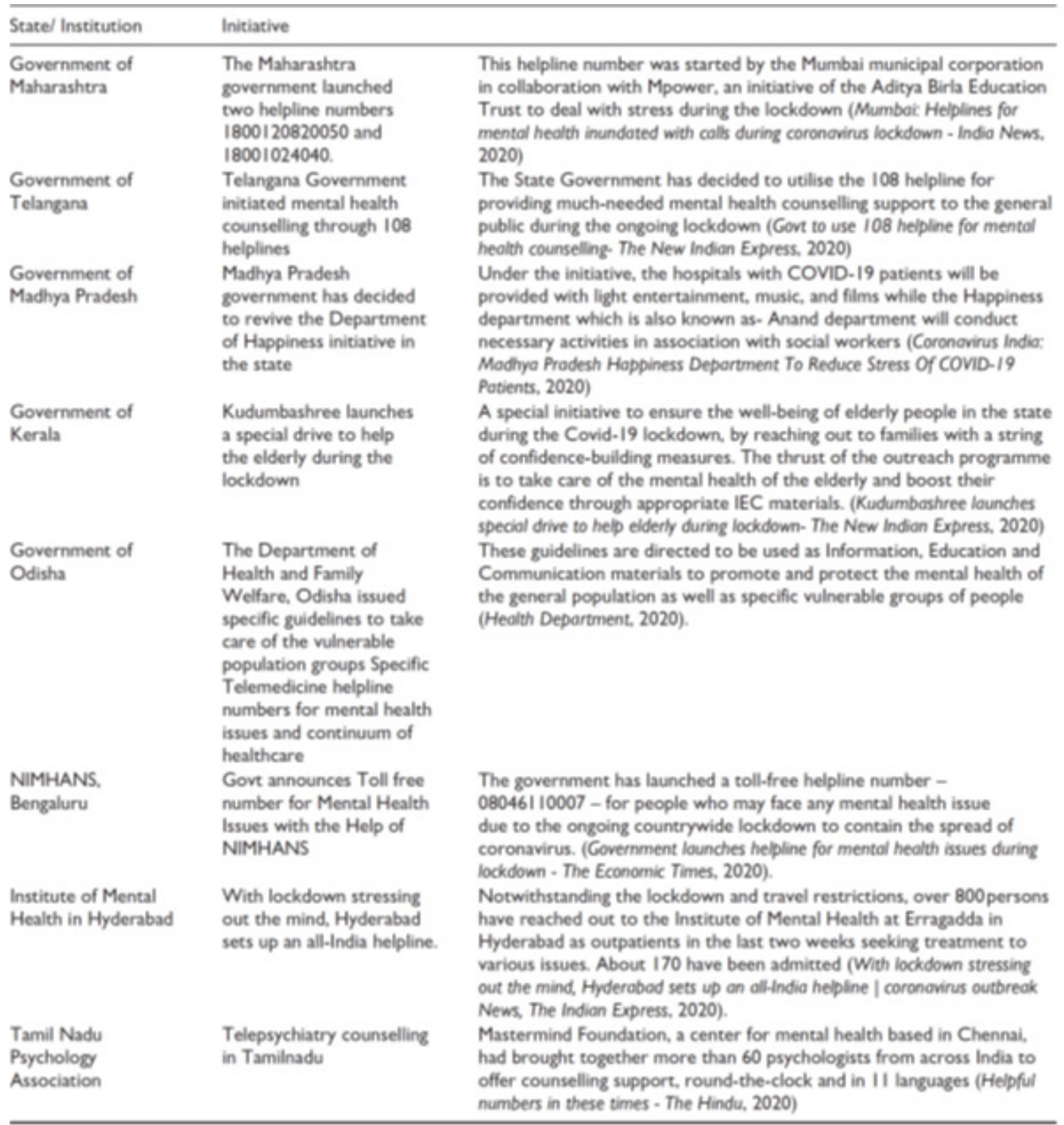

Figure 1: Response to Psychological Issues [4].

\section{Children and Parents}

Many schools and childcare centres across India and around the world have closed down to virtual instruction for at least some time to help slow the spread of coronavirus. These closures cause constant disruption and change in their day-to-day routines for children and their parents. Pand emic research focuses on poor mental health and well-being for children and their parents.

Researchers have found that adults in households with children under 18 have a slightly higher risk for reporting symptoms of anxiety and/or depression than adults in households without ( $45 \%$ vs. $41 \%$, respectively, as of December 2020). In particular, women have been more likely than men to report anxiety and/or depression symptoms during the pandemic in households with children under the age of 18 years (as of December 2020, 49 percent vs. 40 percent, respectively). Similarly, during a pandemic, researchers generally found women are more likely to report negative mental health effects among parents than men [3].

\section{Psychological Issues during COVID in India and Response}

The psychological issues in India were examined by Adrija Roy [4] and Group from India through various publications. According to the study published, stress, anxiety, depression, insomnia, denial, anger and fear were major problems for mental health. One of the vulnerabilities in this regard was children and older people, front-line workers, people with current mental health conditions. Connected suicides with COVID-19 were also more common [4]. 


\section{Epilepsy Foundation experience of Psychological challenges in Indian People with Epilepsy}

The Epilepsy Foundation works in India with the aim of reducing stigma and myths about epilepsy. The Epilepsy Foundation's mission is to offer free medicine to people with epilepsy who are in need. Epilepsy Foundation holds annual camps for people with epilepsy in order to achieve this goal and vision.

From the data Epilepsy Foundation, we evaluated a total of 100 patients on different quality of life components, such energy, work limitations, physical AED problems, COVID parameters for psychological purposes, psychological impact of AEDs, social limitations, memory difficulties, and seizures. Overall QoL, quality of life in distress linked to epilepsy. As regards the response, during this lock-down, people with epilepsy feel more alone. They are significantly suffering from anxiety, depression, seizure worry [5].

\section{Conclusion}

The second wave of COVID has arrived. This ban has been extended for more than a year. People feel isolated, worried about their future health. With the lack of health care, job loss and business cuts, the eco system is stressed. All of these factors influence people's mental, physical and social health. The patients are recovering from the
COVID, however, the infrastructure should be equipped themselves for long term impact on mental health due to COVID. As of now, whatever our experience is, we think this is tip of the iceberg for mental health.

Maintaining a positive approach would help plan and priority the planning and prioritisation of mental health sources in order to address the most fragile, risk communication strategies and the evolving epidemiology of COVID-19.

\section{References}

1. Kumar U (2021) Bidirectional associations between COVID-19 and psychiatric disorder: retrospective cohort studies of 62354 COVID-19 cases in the USA. Lancet 8(12): 130-140.

2. Panchal N (2021) The Implications of COVID-19 for Mental Health and Substance Use. KFF

3. NCHS (2020) National Center for Health Statistics. US: National Center for Health Statistics (NCHS).

4. Roy A (2020) Mental health implications of COVID-19 pandemic and its response in India. International Journal of Social Psychiatry 1-14.

5. Surya N (2020) Quality of Life assessment of people with Epilepsy in COVID Pandemic. International Journal of Psychiatry 5: 1-18. 